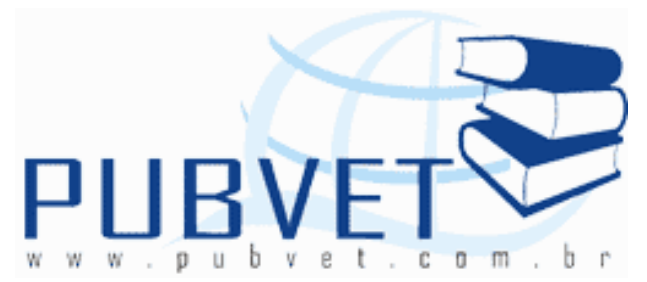

PUBVET, Publicações em Medicina Veterinária e Zootecnia.

\title{
Efeitos da associação maleato de midazolam, citrato de fentanila e cloridrato de cetamina em jibóias Boa constrictor Linnaeus, 1758 (SQUAMATA: BOIDAE)
}

\section{Simone Borges Salgueiro de Simone ${ }^{1}$ e André Luis Quagliatto Santos ${ }^{2}$}

${ }^{1}$ Mestranda em Ciências Veterinárias da Universidade Federal de Uberlândia; ${ }^{2}$ Docente da Faculdade de Medicina Veterinária da Universidade Federal de Uberlândia. Laboratório de Estudo e Pesquisa em Animais Silvestres, Faculdade de Medicina Veterinária, Universidade Federal de Uberlândia (UFU). Rua Piauí, s/n, Bloco 4S, Bairro Jardim Umuarama, Uberlândia, MG, 38400-902, Brasil. *Autor para correspondência: simonebssimone@hotmail.com

\section{Resumo}

A fim de adequar o agente anestésico á rápida indução, com a diminuição do tempo de recuperação e efeitos colaterais, propôs-se testar a associação de cetamina $40 \mathrm{mg} / \mathrm{Kg} / \mathrm{IM}$, midazolan $2 \mathrm{mg} / \mathrm{Kg} / \mathrm{IM}$ e fentanila $0,01 \mathrm{mg} / \mathrm{Kg} / \mathrm{IM}$ em Boa constrictor. Os parâmetros anestésicos foram mensurados através de escores subjetivos durante o trans-anestésico e observou-se também o tempo de duração da anestesia. Constatou-se que em relação ao tempo de permanência em escore três de manipulação, os animais se comportaram de forma semelhante. Todos foram analgesiados, pois não se detectou presença de sensibilidade dolorosa frente à estimulação. Observou-se ausência de tônus da cabeça em todos os espécimes, porém este escore foi atingido em 
SIMONE, S.B.S. e SANTOS, A.L. Efeitos da associação maleato de midazolam, citrato de fentanila e cloridrato de cetamina em jibóias Boa constrictor Linnaeus, 1758 (SQUAMATA: BOIDAE). PUBVET, Londrina, V. 5, N. 21, Ed. 168, Art. 1132, 2011.

diferentes tempos. A perda da capacidade de se locomover foi alcançada por todas as jibóias. Em relação à perda da reação postural de endireitamento os cinco exemplares perderam essa capacidade em 15 minutos após aplicação do fármaco, e o retorno deu-se em média aos 176,66 $\pm 45,09$ em dois animais e os $295 \pm 8,66$ minutos em três. Não houve variação na freqüência cardíaca entre os animais nos 300 minutos de observação e todos os protocolos foram seguros, uma vez que não ocorreram óbitos. A associação proposta promoveu analgesia, relaxamento muscular e anestesia nas serpentes do gênero Boa.

Palavras-chave: Fisiologia, ophidia, répteis, sedação, serpentes.

\title{
Effects of the combination midazolam, fentanyl and ketamine in boas Boa constrictor Linnaeus, 1758 (Squamata Boidae)
}

\begin{abstract}
In order to adapt the anesthetic agent to the rapid induction, with the decrease in recovery time and side effects, we proposed to test the association of ketamine $40 \mathrm{mg} / \mathrm{kg} / \mathrm{IM}$, midazolan $2 \mathrm{mg} / \mathrm{k} / \mathrm{IM}$ and fentanyl $0.01 \mathrm{mg} / \mathrm{kg} / \mathrm{IM}$ in Boa constrictor. The anesthetic parameters were measured using subjective scores during the trans-anesthetic of anesthesia. It was found that in relation to time spent in scoring three of manipulation, the animals behaved similarly. All were anesthetized therefore we not found the presence of soreness before stimulation. We observed lack of tone of the head in all specimens, but this score was reached at different times. At different times of loss of locomotion was achieved by all Boa constrictor. Regarding the loss of postural reaction righting the five copies lost this capacity in 15 minutes after applying the drug, and recurrence occurred in an average of $176.66 \pm 45.09$ in two animals and $295 \pm 8.66$ minutes in three. There was no change in heart rate among animals in 300 minutes of observation and all protocols were safe, since no deaths occurred. The proposed association caused analgesia, muscle relaxation and anesthesia in snakes of the genus Boa.
\end{abstract}

Keywords: Physiology, ophidian, reptiles, sedation, snakes. 
SIMONE, S.B.S. e SANTOS, A.L. Efeitos da associação maleato de midazolam, citrato de fentanila e cloridrato de cetamina em jibóias Boa constrictor Linnaeus, 1758 (SQUAMATA: BOIDAE). PUBVET, Londrina, V. 5, N. 21, Ed. 168, Art. 1132, 2011.

\section{INTRODUÇÃO}

Os ofídios são répteis que habitam principalmente as regiões temperadas e tropicais e por serem ectotérmicos, dependem do calor externo para manterem seu metabolismo ideal (Kolesnikovas et al. 2006). No Brasil, o gênero Boa engloba 17 espécies e se distribui por todo o território nacional, sendo a espécie Boa constrictor a mais encontrada no cerrado e na mata de araucária (Campbell \& Lamar 1989, Borges \& Araujo 1998).

A ausência do diafragma nos répteis implica em uma participação maior de alguns músculos estriados durante a respiração (Bennett 1996). Em planos anestésicos profundos pode-se ter a paralisação destes ocasionando uma parada respiratória (Bennett 1991). Entretanto, são capazes de permanecer por tempo prolongado em apnéia, compensada pela respiração anaeróbica.

Outra característica dos organismos pertencentes à classe Reptilia é a presença do sistema porta-renal capaz de coletar o sangue proveniente da cauda e região pélvica e conduzi-lo aos rins (Hildebrand \& Goslow 2006). Por possuir uma baixa taxa metabólica, relacionada com a temperatura ambiente, ao se administrar agentes anestésicos sistêmicos, as serpentes apresentam tempo de indução e recuperação amplamente variáveis (Hall \& Clarck 1987).

Estudo de parâmetros anestésicos que informam a ação e efeitos colaterais de fármacos necessários à contenção química de répteis tornou-se imprescindível na medicina veterinária, sendo necessários para realização de exames físicos e procedimentos clínicos cirúrgicos. Opióides, barbitúricos, relaxantes musculares e dissociativos têm sido usados para produzir imobilização e sedação dos mesmos (Heaton-Jones 2004).

Diversas drogas apresentam estas características, como a cetamina, anestésico dissociativo que promove efeito de sedação, suficiente para realização de pequenas intervenções como exame clínico, radiológico e sutura de pele (Rang et al. 2004), midazolam, um agente hipnótico, miorrelaxante e depressor do sistema nervoso central, rapidamente absorvido e metabolizado no organismo e a fentanila, opióide e potente analgésico (Tranquilli et al. 
SIMONE, S.B.S. e SANTOS, A.L. Efeitos da associação maleato de midazolam, citrato de fentanila e cloridrato de cetamina em jibóias Boa constrictor Linnaeus, 1758 (SQUAMATA: BOIDAE). PUBVET, Londrina, V. 5, N. 21, Ed. 168, Art. 1132, 2011.

2007). Ainda assim, informações relativas ao emprego de anestésico nestes animais são escassas e, freqüentemente, relatadas de forma inadequada (Bennett 1991).

A escolha da medicação pré-anestésica denota algumas controvérsias em relação ao seu uso de rotina. Alguns autores recomendam o uso de parassimpatolíticos como o sulfato de atropina na dose de $0,04 \mathrm{mg} / \mathrm{Kg}$ intramuscular ou intraperitoneal, para prevenir a secreção excessiva e minimizar o risco de bradicardia após a indução, embora outros autores julguem desnecessário, pois a secreção excessiva no trato respiratório não é comumente visto na anestesia de répteis. Para a atividade antisecretória, o glicopirrolato é preferido, tornando-se o mais eletivo (Schumacher 1996).

Tem-se constatado um crescimento na criação de serpentes como animais exóticos de estimação, sendo cada vez mais necessária a realização de intervenções clínica e cirúrgica. Devido à falta de informações relacionadas com a espécie, erros de manipulação e administração de tais fármacos, tornaram-se comuns (Cooper \& Sainsbury 1997).

Para o sucesso de qualquer procedimento em espécies silvestres é necessário o conhecimento de sua anatomia e fisiologia, especialmente das serpentes, haja vista suas características peculiares (Schumacher 1996), assim objetivou-se avaliar os efeitos da associação cetamina $40 \mathrm{mg} / \mathrm{Kg} / \mathrm{IM}$, midazolan $2 \mathrm{mg} / \mathrm{Kg} / \mathrm{IM}$ e fentanila 0,01mg/Kg/IM em Boa constrictor, afim de minimizar tais equívocos.

\section{MATERIAL E MÉTODOS}

Conduziu-se o experimento no Laboratório de Ensino e Pesquisa em Animais Silvestres (LAPAS) da Universidade Federal de Uberlândia (UFU) com cinco jibóias hígidas, de ambos os sexos, provenientes de apreensão do IBAMA, para realização do protocolo anestésico, segundo a liberação No 092/10 do comitê de ética na utilização de animais. 
SIMONE, S.B.S. e SANTOS, A.L. Efeitos da associação maleato de midazolam, citrato de fentanila e cloridrato de cetamina em jibóias Boa constrictor Linnaeus, 1758 (SQUAMATA: BOIDAE). PUBVET, Londrina, V. 5, N. 21, Ed. 168, Art. 1132, 2011.

Os espécimes foram pesados em uma balança simples (Modelo 9094, Toledo, São Bernardo do Campo, SP, Brasil) com peso médio de $1,580 \mathrm{Kg}$ e acondicionados individualmente em caixas plásticas numeradas sequencialmente a fim de identificá-las.

Aferiu-se a freqüência cardíaca (FC) de todos os exemplares com o aparelho doppler vascular (Modelo DV-20, Microem, Ribeirão Preto, SP, Brasil), e mediante prévia anti-sepsia com álcool iodado submeteu-os a associação cetamina $40 \mathrm{mg} / \mathrm{Kg} / \mathrm{IM}$, midazolan $2 \mathrm{mg} / \mathrm{Kg} / \mathrm{IM}$ e fentanila $0,01 \mathrm{mg} / \mathrm{Kg} / \mathrm{IM}$ com auxílio de seringas estéreis de $3 \mathrm{~mL}$, acopladas a agulhas hipodérmicas $13 \mathrm{x}$ 0,45 mm (Med-Hut, Curitiba, PR, Brasil) por via intramuscular profunda na musculatura do dorso na metade cranial do corpo, segundo Cubas et al. (2007). Os fármacos foram administrados com intervalos de 10 minutos entre uma aplicação e outra.

Durante o período trans anestésico, as jibóias foram avaliadas em relação aos parâmetros anestésicos estipulados, como locomoção, tônus muscular, tônus da cabeça, manipulação, estímulos dolorosos (superficial e profundo), frequencia cardíaca, reação postural de endireitamento e grau de sedação, segundo a metodologia empregada por Malley (1997).

1. Redução satisfatória da atividade locomotora: capacidade do animal em se locomover, identificada como normal (1), difícil (2) ou ausente (3).

2. Tônus muscular: conseguir manter o corpo em sua postura normal: normal (1), difícil (2) ou ausente (3).

3. Tônus da cabeça: Capacidade de manter a cabeça ereta: normal (1), difícil (2) ou ausente (3).

4. Facilidade de manipulação: aceitação frente à abertura da boca, provocada pelo manipulador: normal (1), difícil (2) ou ausente (3).

5. Resposta ao estímulo doloroso: através da estimulação por agulha hipodérmica ao longo do corpo do animal. 
SIMONE, S.B.S. e SANTOS, A.L. Efeitos da associação maleato de midazolam, citrato de fentanila e cloridrato de cetamina em jibóias Boa constrictor Linnaeus, 1758 (SQUAMATA: BOIDAE). PUBVET, Londrina, V. 5, N. 21, Ed. 168, Art. 1132, 2011.

6. Freqüência cardíaca: número de batimentos cardíacos por minuto, com intervalos regulares a cada 15 minutos até o retorno as condições préanestésicas.

7. Perda da reação postural de endireitamento (RPE): avaliado quanto à dificuldade de retornar espontaneamente à posição ventro-dorsal.

8. Retorno da RPE: readquire a capacidade de retorno espontâneo à posição ventro-dorsal.

Aferiram-se os cinco primeiros parâmetros anestésicos descritos em intervalos regulares a cada 15 minutos até o retorno as condições préanestésicas.

Alterações na locomoção foram avaliadas através de estímulo manual, capaz de promover movimentos de serpentear, sinais estes que ausentes estipularam a presença de relaxamento muscular. $O$ uso de agulhas hipodérmicas como instrumento de estimulação ao longo do corpo do animal permitiu observar presença ou ausência de sensibilidade dolorosa.

Considerou-se em estado de anestesia as serpentes que apresentaram redução satisfatória da atividade locomotora, relaxamento muscular e da cabeça, facilidade de manipulação e perda de sensibilidade, simultaneamente.

A cada hora, a temperatura ambiente foi registrada com a utilização de termohigrômetro digital (Cristália, Itapira, SP, Brasil), onde a média foi de $28^{\circ} \mathrm{C}$, estando dentro da faixa de conforto térmico das jibóias, ou seja, entre 25 e $30^{\circ} \mathrm{C}$, segundo Mosley (2005) e Tranquilli et al.(2007) sendo esperada a melhor ação do fármaco utilizado.

Avaliaram-se os resultados através de análise descritiva.

\section{RESULTADOS}

Três animais não se diferenciaram em relação ao tempo em que perderam a capacidade de locomoção, pois 15 minutos após a aplicação da cetamina eles atingiram o escore três para este parâmetro. Um espécime atingiu este escore após 30 minutos e outro após 45 minutos. As serpentes 
SIMONE, S.B.S. e SANTOS, A.L. Efeitos da associação maleato de midazolam, citrato de fentanila e cloridrato de cetamina em jibóias Boa constrictor Linnaeus, 1758 (SQUAMATA: BOIDAE). PUBVET, Londrina, V. 5, N. 21, Ed. 168, Art. 1132, 2011.

obtiveram permanência maior em escore três e menor no dois. Esse fato ocorreu devido à breve passagem de dois animais pelo escore dois e a passagem direta para o escore três de alguns animais. Três jibóias retornaram sua locomoção no fim dos 300 minutos de avaliação. As outras duas regressaram para este escore nos 120 e 240 minutos respectivamente.

$\mathrm{Na}$ avaliação do tônus muscular observou-se que dois animais passaram diretamente do escore um para o três no primeiro tempo de avaliação e permaneceram neste escore durante o experimento. Um espécime não atingiu o escore máximo permanecendo em escore dois durante o tempo de observação e manteve-se nesta condição durante os 300 minutos. Por fim, duas jibóias tiveram perda gradativa do tônus muscular, no qual passaram do escore um para o dois até atingir a perda deste parâmetro, porém retornaram apenas ao escore dois no final da avaliação.

A ausência do tônus da cabeça ocorreu de forma semelhante em três animais, estes atingiram o escore dois após 30 minutos da aplicação do último fármaco, no entanto, diferiram no tempo de retorno, o qual retornaram ao escore dois aos 120, 270 e 300 minutos. Duas jibóias tiveram uma passagem direta para o escore três desta avaliação, e retornaram aos 270 e 300 minutos respectivamente.

Todos os espécimes permaneceram em escore três para manipulação, o que os diferiu foi o tempo que cada animal levou para atingir este escore, sendo que três animais alcançaram este escore em 15 minutos e dois em 30 minutos. Quatro animais mantiveram-se em escore três de manipulação nos 300 minutos de avaliação e apenas um retornou ao escore dois aos 270 minutos.

Durante a avaliação da presença ou ausência de sensibilidade dolorosa notou-se presença de analgesia no máximo 30 minutos após a administração da cetamina. A sensibilidade retornou aos 165, 195 e 285 minutos em três animais e nos 300 minutos em dois.

Em relação à perda da reação postural de endireitamento, as jibóias se comportaram de forma semelhante, todas perderam a capacidade de retorno 
SIMONE, S.B.S. e SANTOS, A.L. Efeitos da associação maleato de midazolam, citrato de fentanila e cloridrato de cetamina em jibóias Boa constrictor Linnaeus, 1758 (SQUAMATA: BOIDAE). PUBVET, Londrina, V. 5, N. 21, Ed. 168, Art. 1132, 2011.

em sua posição normal 15 minutos em média após a aplicação da cetamina. No entanto, o tempo de retorno da reação postural de endireitamento foi diferente para alguns animais, dois retornaram em média aos 176,66 $\pm 45,09$ minutos pós-injeção do último fármaco e três não readquiriram esta capacidade antes dos $295 \pm$ 8,66 minutos de observação.

Não houve variação na freqüência cardíaca entre os animais nos 300 minutos de observação. A via de administração adotada e todos os protocolos foram seguros, uma vez que não ocorreram óbitos. Todos os animais foram considerados anestesiados.

\section{DISCUSSÃO}

Devido às particularidades anatômicas e comportamentais, a anestesia em serpentes deve priorizar 0 uso de medicamentos passíveis de administração intramuscular e elevada segurança anestésica (Bennett 1991). Seguindo as indicações de Bennett (1991) e Hildebrand \& Goslow (2006) a fim de evitar a rápida eliminação dos fármacos optou-se pela aplicação intramuscular no terço proximal cranialmente aos rins, devido à presença do sistema porta-renal, capaz de coletar o sangue proveniente da cauda e região pélvica e conduzi-lo aos rins antes de passar pela circulação geral (Hall \& Clarck 1987).

De acordo com Bennett et al. (1998), a indução anestésica compreende o período entre a administração do agente e a perda da reação postural de endireitamento (RPE). Para quatro animais esse período foi compreendido entre 14 e 30 minutos e um espécime alcançou este estágio em apenas cinco minutos. Mesmo havendo variações no tempo de indução a utilização do benzodiazepínico como MPA se mostrou eficaz, pois promoveu rápida sedação associada a um bom relaxamento muscular, efeitos propostos pelo midazolam.

Para a constatação da indução e recuperação da anestesia foram avaliados tônus de cabeça, tônus muscular, reação postural de endireitamento e locomoção (Malley 1997), aferidos em minutos até a perda total da variável. 
SIMONE, S.B.S. e SANTOS, A.L. Efeitos da associação maleato de midazolam, citrato de fentanila e cloridrato de cetamina em jibóias Boa constrictor Linnaeus, 1758 (SQUAMATA: BOIDAE). PUBVET, Londrina, V. 5, N. 21, Ed. 168, Art. 1132, 2011.

Para isso, os parâmetros foram analisados imediatamente após a adiministração dos fármacos estendendo-se até o tempo máximo proposto de 300 minutos. Pela ausência de dados na literatura consultada, referentes à avaliação anestésica para os parâmetros citados, considerou-se a associação em questão eficiente, pois se constatou efeito ótimo para todas as serpentes nestes quesitos.

Anestesia é o tempo entre a perda e o retorno da RPE (Bennett et al. 1998). Em conformidade com este conceito, duas jibóias voltaram da anestesia aos $176,66 \pm 45,09$ minutos. Esse parâmetro não foi observado antes dos 295 $\pm 8,66$ minutos em três animais. Fato explicado não só pela escolha do dissociativo como agente anestésico, mas também pela sua associação com benzodiazepínicos, que favorece na indução, porém pode provocar períodos de recuperação bastante prolongados (Rojas 2002).

O ponto primário da anestesia é tornar o animal insensível à dor (Steffey 2003), logo, considera-se que os espécimes entraram em plano anestésico ideal, pela ausência de sensibilidade aos estímulos provocados ao longo do corpo do animal já no primeiro tempo de avaliação. Esta ausência deve-se a associação anestésica realizada. Neste sentido, o uso da fentanila se destaca como analgésico, pois se estima que seja 80 vezes mais potente que a morfina promovendo melhor analgesia (Malley 1997, Bennett 1998, Mosley 2005).

Sendo a recuperação compreendida entre o retorno da RPE e das condições pré-anestésicas (Bennett et al. 1998), observou-se que, três serpentes se recuperaram antes dos 300 minutos de administração dos fármacos e duas após este tempo.

Apesar de a literatura citar alguns estudos com o uso isolado de cetamina em serpentes, ainda há divergência sobre a dose ideal desse anestésico para uma contenção química apropriada. Glenn et al. (1972) determinaram a DL50 da cetamina para serpentes da espécie Crotalus atrox como $154 \mathrm{mg} / \mathrm{kg}$ e que doses entre $55-88 \mathrm{mg} / \mathrm{kg}$ promoveram uma contenção química adequada para a realização de pequenos procedimentos. Outros artigos também citam doses entre $50-80 \mathrm{mg} / \mathrm{kg}$ como adequadas para 
SIMONE, S.B.S. e SANTOS, A.L. Efeitos da associação maleato de midazolam, citrato de fentanila e cloridrato de cetamina em jibóias Boa constrictor Linnaeus, 1758 (SQUAMATA: BOIDAE). PUBVET, Londrina, V. 5, N. 21, Ed. 168, Art. 1132, 2011.

contenção (Cooper 1974, Green et al. 1981, Hill \& Mackessy 1997) com período de latência de até 30 minutos. Como os agentes dissociativos promovem discreta analgesia visceral (Bennett 1991), o uso de cetamina isolada deve ser realizado apenas para contenção física ou procedimentos cirúrgicos cutâneos. Para procedimentos intracelomáticos, o ideal é a associação desse anestésico com opióides, anti-inflamatórios não esteróides ou agonistas alfa-2 adrenérgicos, a fim de promover melhor analgesia (Malley 1997, Bennett 1998, Mosley 2005). No entanto, o uso da cetamina na dose de $40 \mathrm{mg} / \mathrm{kg}$ associada com fentanila na dose de $0,01 \mathrm{mg} / \mathrm{kg}$ e midazolam na dose de $2 \mathrm{mg} / \mathrm{kg}$ em Boa Constrictor, mostrou-se eficaz, visto que os animais apresentaram perda da capacidade de locomoção, relaxamento muscular satisfatório, facilidade de manipulação e ausência de sensibilidade dolorosa, podendo ser utilizada em vários procedimentos.

Carregaro (2009), avaliando tônus muscular, tônus de cabeça e reação postural de endireitamento em cascavéis anestesiadas com cetamina a $80 \mathrm{mg} / \mathrm{kg}$ e as submetendo a variações de temperatura, observou que em hipotermia o período de indução provocado pela cetamina foi semelhante em todos os animas. Todavia, na temperatura de conforto a reação postural de endireitamento foi abolida antes dos demais. O período de recuperação ao se observar as três variáveis foram iguais entre si, entretanto em hipotermia este período foi superior. Ao se trabalhar com cetamina a $40 \mathrm{mg} / \mathrm{kg}$ associada com midazolam e fentanila sem variar a temperatura, mantendo todas as jibóias em temperatura ideal pode-se observar que o período de indução não variou quando se avaliou locomoção entre os animais, mas o tônus muscular e o tônus da cabeça diferiram entre eles.

A associação de cetamina na dose de 20 a $40 \mathrm{mg} / \mathrm{Kg}$ e midazolam na dose de $2 \mathrm{mg} / \mathrm{Kg}$ provocou sedação e bom relaxamento muscular em tartarugas (Schumacher 1996). Tal protocolo anestésico administrado unicamente pela via intramuscular pode revelar-se satisfatório em répteis não debilitados, mas a analgesia e relaxamento muscular não são ideais (Cooper \& Sainsbury 1997). Haja vista, que neste estudo, preconizaram-se as mesmas 
SIMONE, S.B.S. e SANTOS, A.L. Efeitos da associação maleato de midazolam, citrato de fentanila e cloridrato de cetamina em jibóias Boa constrictor Linnaeus, 1758 (SQUAMATA: BOIDAE). PUBVET, Londrina, V. 5, N. 21, Ed. 168, Art. 1132, 2011.

doses citadas, porém, os resultados diferiram. Todas as jibóias obtiveram um relaxamento muscular satisfatório e uma sedação adequada.

Agentes anestésicos inalatórios como éter, clorofórmio (Brazenor \& Kaye 1953) e fluotano (Hackenbrock \& Finster 1963) são convenientes para o uso em répteis, principalmente serpentes. Com estes agentes, no entanto, a duração e a profundidade da anestesia são de difícil controle. Halpern \& Frumin (1973), Halpern \& Silfen (1974), Halpern (1974, 1976a e 1976b) ao pesquisarem a ação do fluotano em Thamnophis sirtalis e $T$. radix, relatam o sucesso de sua utilização em procedimentos de curta duração, pois seu efeito é de 5 a 10 minutos, porém, quando se aprofunda a anestesia para aumentar sua duração, a taxa de mortalidade fica em torno de 33\%. Já a associação proposta teve uma duração satisfatória sem nenhum índice de mortalidade.

Nembutal sódio (Betz 1962), tiopental sódio (Karlstrom \& Cook 1955) e cetamina (Glenn et al. 1972a), drogas anestésicas usadas freqüentemente em répteis, requerem um tempo de indução de cerca de 30 minutos e o efeito anestésico permanece por vários dias a uma semana. No entanto, na dose de $40 \mathrm{mg} / \mathrm{kg}$ de cetamina, dois animais saíram do plano anestésico ideal em 195 e 270 minutos, respectivamente, e os outros após os 300 minutos de avaliação, diferindo do tempo encontrado pelos autores.

A dose de $80 \mathrm{mg} / \mathrm{kg}$ de cetamina utilizada por Carregaro et al. (2009) proporcionou imobilização dos animais com um período de indução em torno de 20 minutos, em todo os animais, mesmo os submetidos a hipotermia. Outros estudos observaram um período de indução superior a 30 minutos em serpentes anestesiadas com cetamina e mantidas em temperatura ambiente de $22^{\circ} \mathrm{C}$, o que reforça ainda mais essa hipótese (Glenn et al. 1972 , Green et al. 1981). Os dados encontrados corroboram com os deste estudo onde o período de indução foi de 15 minutos em uma zona de conforto térmico.

Acredita-se que, uma variação na temperatura corpórea mais acentuada poderia evidenciar diferenças de absorção pelos animais, conforme demonstraram Stirl et al. (1996), no uso de tiletamina/zolazepam em Boa constrictor. Por serem animais ectotérmicos, a temperatura corporal exerce 
SIMONE, S.B.S. e SANTOS, A.L. Efeitos da associação maleato de midazolam, citrato de fentanila e cloridrato de cetamina em jibóias Boa constrictor Linnaeus, 1758 (SQUAMATA: BOIDAE). PUBVET, Londrina, V. 5, N. 21, Ed. 168, Art. 1132, 2011.

efeito decisivo na cinética da maioria dos medicamentos administrados nesta classe. Situações de hipotermia promovem redução na biotransformação dos fármacos, alterando o período de ação e recuperação (Mosley 2005). Deste modo, recomenda-se que os animais sejam mantidos aquecidos tanto durante o processo anestésico como no período de recuperação (Malley 1997, Bennett 1998). Todavia, o aquecimento não deve ser excessivo, uma vez que pode ser deletério por elevar demasiadamente o metabolismo. Assim, para os répteis terrestres, o recomendável é a manutenção da temperatura ambiente entre 25 e $30^{\circ} \mathrm{C}$ (Bennett 1991, Divers 1996). Esperando-se o melhor efeito dos fármacos os animais foram mantidos em um ambiente fechado sem grandes alterações de temperatura, a qual se manteve em média $28^{\circ} \mathrm{C}$.

Em situações de hipotermia os répteis apresentam diminuição da FC, porém mantém o débito cardíaco devido uma compensação do volume sistólico e vasoconstrição periférica (Bennett 1991, Carregaro et al. 2009). Estudos relataram aumento na FC após a administração intramuscular de cetamina em serpentes (Stirl et al. 1996, Schumacher et al. 1997) e apenas doses acima de $110 \mathrm{mg} / \mathrm{kg}$ produzem bradicardia e bradipnéia, instaurando-se o quadro em até 20 minutos (Glenn et al. 1972, Bennett 1991, Sedgwick \& Borkowski 1996). Tais dados confirmam os aqui encontrados, pois das serpentes avaliadas somente uma teve sua FC reduzida após a aplicação do fármaco, no entanto essa diferença não foi estatisticamente significativa.

Optou-se por medir a freqüência respiratória ao invés do tempo de apnéia, pois os répteis podem permanecer assim por até 24 horas realizando respiração anaeróbica e percutânea (Bennett 1991). Ainda assim o método de mensuração foi eficiente, onde os animais apresentaram de um a dois movimentos respiratórios por minuto a cada 15 minutos de observação, mantendo o mesmo padrão durante todo o experimento. Estes achados concordam com os de Carregaro et al. (2009), que observou o tempo de apnéia de Crotalus durissus e relatou que em temperatura normal este tempo não diferiu entre si. Destaca-se ainda a ação depressora da cetamina sobre a respiração nestes animais (Glenn et al. 1972, Bennett 1991, Sedgwick \& 
SIMONE, S.B.S. e SANTOS, A.L. Efeitos da associação maleato de midazolam, citrato de fentanila e cloridrato de cetamina em jibóias Boa constrictor Linnaeus, 1758 (SQUAMATA: BOIDAE). PUBVET, Londrina, V. 5, N. 21, Ed. 168, Art. 1132, 2011.

Borkowski 1996), elevando- se o tempo de apnéia em ambos os grupos após 30 minutos, apesar de não diferirem em relação ao basal.

\section{CONCLUSÕES}

A associação fentanila na dose de $0,01 \mathrm{mg} / \mathrm{kg}$ com midazolan na dose de $2 \mathrm{mg} / \mathrm{kg}$ e cetamina na dose de $40 \mathrm{mg} / \mathrm{kg}$, promoveu analgesia, relaxamento muscular e anestesia nas serpentes do gênero Boa. Mostrou-se eficiente para realização de procedimentos prolongados e dolorosos, no entanto, os animais devem permanecer em sua temperatura de conforto, para que não haja alterações no seu metabolismo e obtenham uma boa recuperacão anestésica.

\section{REFERÊNCIAS}

Bennett R.A.1991. A review of anesthesia and chemical restraint in reptiles.

Journal of Zoo and Wildlife Medicine. 22 (3): 282-303.

Bennett R.A. 1996. Anesthesia, p. 241-247 In: Marder, D.R. (Ed.), Reptile Medicine and Surgery. W.B. Saunders, Philadelphia.

Bennett R.A., Schumacher, J., Hedjazi-Haring, K., Newell, S.M. 1998. Cardiopulmonary and Anesthetic Ef fects of Propofol Administered Intraosseously to Green Iguanas. Journal of American Veterinary Medical Association. 212 (1): 93-98.

Betz T.W. 1962. Surgical anesthesia in reptiles, with special references to the water snakes, Natrix rhombifera. Copeia. 1962(2): 285-287.

Borges R.C., Araujo, A.F.B. 1998. Seleção de hábitat em duas espécies de jararaca (Bothrops moojeni Hoge e B. neuwiedi Wagler). Revista Brasileira de Biologia. 58 (4): 591-601.

Brazenor C.W., \& G. Kaye. 1963. Anesthesia for reptiles. Copeia 1953:165-170.

Campbell J.A., Lamar W.W. 1989. The Venomous Reptiles of Latin America. Ithaca, Londres. $425 p$.

Carregaro A. B., Cruz M. L., Cherubini A. L., Luna S. P.L. 2009. Influência da temperatura corporal de cascavéis (Crotalus durissus) submetidas à anestesia com cetamina. Pesq. Vet. Bras. 29 (12):969-973.

Cooper J.E., Sainsbury A.W. 1997. Perguntas e Respostas Ilustradas de Medicina Veterinária: Espécies exóticas. Manole, São Paulo. 168 p.

Cubas Z.S., Silva J.C.R., Catao-Dias J.L. 2007.Tratado de animais selvagens. Medicina Veterinaria. Editora Roca, São Paulo. 1354 p.

Divers S. 1996. Basic reptile husbandry, history taking and clinical examination. In: Practice. 18:51-65. 
Glenn J.L., Straight R. \& Snyder C.C. 1972. Clinical use of ketamine hydrochloride as an anesthetic agent for snakes. Am. J. Vet. Res. 33:1901-1903.

Glenn, J., R. Straight \& C. C. Snyder. 1972a. Ketalar-a new anesthetic for use in snakes. Internat. Zoo. Yearbook, London. 12:224-226.

Green C.J., Knight J., Precious S. \& Simpkin S. 1981. Ketamine alone and combined with diazepam or xylazine in laboratory animals: a 10 year experience. Laboratory Animals. 15:163-170

Hackenbrock C.A. \& Finster M. 1963. A rapid and safe inhalation anesthetic for $\cdot$ ious snakes. Copeia. 61 (2): 440-44.

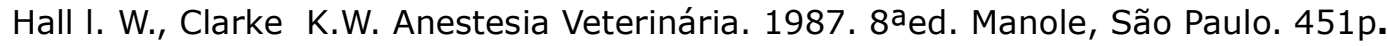

Halpern M. \& N. Frumin. 1973. Retinal projections in a snake, Thamnophis sirtalis. J. Morphol. 141: 359-381.

Halpern M. 1974. Experimental demonstration of the fornix system in a snake. Abst. $4^{a}$ ed. Meet-ing Soc. for Neuroscience. 244

Halpern M., \& R. Silfen. 1974. The efferent con-nections of the nucleus sphericus in the garter snake, Thamnophis sirtalis. Anat. Rec. 178:368.

Halpern M. 1976a. Efferent connections of the lateral and dorsal cortices of snakes of the genus Tham-nophis. Anat. Rec. 184:421.

Halpern M. 1976b. The efferent connections of the olfactory bulb and accessory olfactory bulb in the snakes, Thamnophis sirtalis and Thamnophis radix. J. Morphol. 150:553-578.

Heaton-Jones T.G. 2004. Anestesia e Analgesia de Répteis. In: Greene S.A. Segredos em Anestesia Veterinária. Artmed, Porto Alegre. 47: 349-360.

Hildeband M., Goslow G.E. 2006. Análise da estrutura dos vertebrados. $2^{a}$ ed. Atheneu, São Paulo. 637p.

Hill R.E. \& Mackessy S.P. 1997. Venom yields from several species of colubrid snakes and differential effects of ketamine. Toxicon 35:671-678.

Karlstrom E. L., Ands. F., Cook JR. 1955. Notes on snake anesthesia. Copeia. 1955:57-58.

Kolesnikovas C. K.M., Grego K.F., Albuqerque L.C.R. Ordem Squamata - Subordem Ophidia (Serpente): In: Cubas Z.S., Silva J.C.R.

Malley D. 1997. Reptile anaesthesia and the practicing veterinarian. In: Practice 19:351-368.

Mosley C.A.E. 2005. Anesthesia and analgesia in reptiles. Seminars in Avian and Exotic Pet Medicine.14 (4): 243-262.

Rang, H.P., Dale M.M., Ritter, J.M. 2004. Farmacologia: Elsevier, Rio de Janeiro. 920p.

Rojas L. 2002. Anestesia em reptiles. Boletín GEAS. 3(4): 34-37. Dispor'man em <http://issuu.com/vetsvidasilv/docs/bgeas200203?mode=embed\&documentId=081 $20 \quad 40$ 354-14b5753634a64e1d91e108a8503f0494\&layout=grey>. Acesso em: 24 jul. 2010.

Schumacher J. 1996. Reptiles and Anphibians, p.670-685. In: Thurmon J.C., Tranquilli W.J. Lumb \& Jones Veterinary Anesthesia. Saunders, Pennsylvania.

Sedgwick C.J. \& Borkowski R. 1996. Allometric scaling. Extrapolating treatment regimens for reptiles, p.235-240. In: Mader D.R. (Ed.), Reptile Medicine and Surgery. Saunders Elsevier, Philadelphia. 512p.

Steffey E. P. 2003. Drogas que agem no sistema nervoso central, p.127-143. In: Adams H.R. (Ed), Farmacologia e Terapêutica em Veterinária. $8^{a}$ ed. Guanabara Koogan, Rio de Janeiro. 
Stirl R., Krug P. \& Bonath K.H. 1996. Tiletamine/zolazepam sedation in Boa constrictor and its influence on respiration, circulation and metabolism. First Scientific Meeting European Association of Zoo and Wildlife Veterinarians European Association of Zoo- and Wildlife Veterinarians (EAZWV), Rostock, Germany. p.16-18.

Tranquilli W.J., Thurmon J.C., Grimm, K.A. 2007. Veterinary Anesthesia and Analgesia. Blackwell Publishing. Iowa. 1096p. 